\title{
Maestría en Docencia de la Matemática
}

\author{
El método de análisis - síntesis en la resolución de problemas \\ aritméticos escolares de enunciado verbal
}

\author{
Orlando Rafael Barrios Bustillo
}

Palabras clave: Análisis, Síntesis, Heurística, Problema Aritmético de Enunciado Verbal, Currículo Propuesto, Currículo Logrado, Representación Discursiva del Razonamiento, Representación Esquemática del Razonamiento.

\section{Descripción}

Se reporta a través de este documento un estudio desarrollado en el Colegio Italiano Leonardo da Vinci en relación con una adaptación escolar del método de análisis - síntesis que se utiliza en el tratamiento de los problemas aritméticos de enunciado verbal.

El objetivo principal de este estudio consiste en la identificación y caracterización de las relaciones entre el currículo propuesto y el currículo logrado con respecto a la temática seleccionada. En el proceso, se revisan referentes conceptuales e investigativos sobre la resolución de problemas y sobre el análisis y la síntesis en algunos contextos en donde ha aparecido a través de la historia. Como conclusiones, se emiten las relaciones entre los dos niveles de desarrollo curricular y se formulan consideraciones acerca de cómo podrían reorientarse o complementarse las acciones educativas, para que la utilización del método analítico - sintético sea más significativa en el contexto de la resolución de problemas aritméticos de enunciado verbal.

\section{Contenidos}

La resolución de problemas en Matemáticas es una fuente productora de conocimiento matemático. Este conocimiento se determina a partir de los tipos de problemas que se resuelven, en tanto proveen distintos contextos de experiencia, y de las heurísticas construidas para la resolución de los mismos, en tanto articulan y caracterizan la actividad matemática de los resolutores, estudiantes, en el proceso de resolución.

El método de análisis y síntesis aparece en la historia de las ciencias en diferentes momentos. Es sistematizado inicialmente en Grecia clásica en los Elementos de Euclides en el ámbito de la resolución de problemas en geometría. Pappus describe de manera detallada el análisis y la síntesis y diferencia 
diferentes tipos de análisis. Posteriormente, estas realizaciones de Pappus son retomadas por Descartes, Newton y Riemann, entre otros, cada uno de los cuales propone adaptaciones del método, que permiten ampliar los contextos en donde el análisis y la síntesis son aplicables identificando las condiciones que deben tener los problemas, sistemas o fenómenos para que dicha aplicación sea pertinente.

El método de análisis - síntesis en la resolución de problemas aritméticos aparece reportado en el ámbito escolar en investigaciones soviéticas desarrolladas entre los años sesenta y setenta. Estos estudios se fundamentaron en una postura psicológica desde la cual se considera al pensamiento humano como analítico y sintético. Así pues, el método III que se describe en la historia de las Matemáticas y de otras ciencias incursionó en la escuela convirtiéndose en una heurística para la resolución de problemas.

En el Colegio Italiano Leonardo da Vinci, el método es utilizado desde los años noventa, consolidándose como uno de los principales objetos de aprendizaje de los estudiantes, considerados en el currículo propuesto de matemáticas en la escuela primaria.

En la actualidad, los estudiantes muestran algunas dificultades en el trabajo en la resolución de problemas aritméticos que, en su mayoría, se remiten a la utilización y comprensión de los elementos propios del método. Tales dificultades inciden en la concepción de los estudiantes sobre la resolución de problemas matemáticos en general y en la actividad resolutoria misma. No obstante, los estudiantes han asimilado un método que difiere del propuesto desde la teoría y que permite cuestionar la validez o pertinencia de los que se presume desde el currículo propuesto.

Este documento se encuentra dividido en varios capítulos. El capítulo 1, Aspectos generales de la investigación, contiene la información relativa a los antecedentes y justificación del estudio, a la formulación del problema y descripción de las intenciones de estudio, y a los elementos metodológicos que orientaron el desarrollo del estudio. En el capítulo 2, Elementos conceptuales del marco de interpretación del estudio, se hace un recorrido por los principales referentes conceptuales que constituyen un marco teórico con el cual interpretar la actividad de los estudiantes en torno a la resolución de PAEV con el método de análisis - síntesis. El capítulo 3, Descripción y aplicación de los instrumentos de recolección de la información, contiene la información relativa a las pruebas aplicadas para recoger la información, a la población seleccionada para el desarrollo de las pruebas y a la actividad de los estudiantes frente a las pruebas, conteniendo, además, las justificaciones de las decisiones metodológicas y análisis previos vía a la caracterización del currículo logrado para el establecimiento de relaciones con el propuesto. El capítulo 4, Caracterización del currículo logrado con respecto al método de análisis - síntesis en la resolución de PAEV, se presenta un resumen y ampliación de los análisis sobre los IV resultados de los estudiantes en las pruebas y de las intervenciones en la entrevista semiestructurada, con el objetivo de caracterizar el currículo logrado. Por último, el capítulo 5, Conclusiones, contiene los resultados del estudio. 


\section{Metodología}

El diseño metodológico atiende al objetivo de identificar y clasificar las relaciones entre el currículo propuesto y el currículo logrado en torno al uso del método de análisis - síntesis en la resolución de problemas aritméticos de enunciado verbal. En un primer momento se realizaron revisiones y estudios documentales. Así, inicialmente, se revisaron documentos institucionales, conceptuales y operativos, que permitieron describir y caracterizar el currículo propuesto en torno a la temática de estudio; otra revisión bibliográfica permitió establecer los elementos conceptuales a partir de los cuales fue posible modelar la temática de estudio, construir los instrumentos de recolección de la información y consolidar un marco de interpretaciones sobre la actividad de los estudiantes con el objetivo de caracterizar el currículo propuesto. Tal marco de interpretaciones se consolidó en un contexto de significación a posteriori del trabajo de los estudiantes sobre las pruebas diseñadas y de las intervenciones en la entrevista aplicada. La selección de los estudiantes para las pruebas obedeció a una serie de criterios sobre el desempeño.

\section{Conclusiones}

El estudio muestra que el currículo logrado difiere sustancialmente del currículo propuesto en relación con el uso del método de análisis - síntesis en la resolución de problemas aritméticos de enunciado verbal (PAEV) en el Colegio Italiano Leonardo da Vinci. Con frecuencia, los estudiantes utilizan estrategias que les ayudan a completar los pasos requeridos desde el método, pero que van en contravía de la naturaleza del mismo; así, por ejemplo, ante problemas de una estructura diferente a la que usualmente trabajan, los estudiantes utilizan síntesis parciales antes de representar el razonamiento analítico y la analogía, evocando modelos de problemas ya resueltos para acomodarlos y complementarlos en el proceso de resolución del problema que se les solicita. El objetivo educativo que se formula desde el programa de Matemáticas en la institución tiene que ver con la habilidad de resolver problemas matemáticos; sin embargo, en lo que refiere a los PAEV, los estudiantes fácilmente interpretan la aplicación del método como el objetivo principal de la actividad y, de hecho, a partir de lo indagado, parecería que el camino seleccionado para orientar la resolución de problemas, termina siendo la meta del aprendizaje. 


\section{Ambientes de Aprendizaje y Cultura Estadística a través de un Experimento de Enseñanza para estudiantes de grado noveno}

Ingrith Yadira Álvarez Alfonso

\section{Diana Milena Montoya Cortés}

\section{Contenidos}

El documento en tres secciones muestra el proceso investigativo: la primera parte presenta el planteamiento del problema de investigación, su descripción, formulación y antecedentes (Cap. 1), la justificación (Cap. 2) y los objetivos (Cap. 3), lo que conlleva a formular la segunda sección y fundamentar la investigación en un marco teórico que confluyen en la Cultura Estadística, los Ambientes de Aprendizaje y la Educación Estadística Crítica (Cap. 4) concretadas mediante el Experimento de Enseñanza como metodología de investigación (Cap. 5), lo que permite formular la tercera sección, que presenta el desarrollo del proyecto de investigación (Cap. 6), conduciendo a conclusiones y consideraciones finales.

\section{Metodología}

El Experimento de Enseñanza se desarrolla en cuatro fases, la Fase Preliminar (FP) caracteriza el macro y micro contexto de la clase de noveno grado, generando las condiciones iniciales para la Fase 1 (F1) donde se propone, el Diseño Instruccional y Planeación, desde las Metas de Aprendizaje, la Hipótesis de Investigación y la Trayectoria Hipotética de Aprendizaje. Luego se desarrolla el Experimento en el Aula (F2), donde se describen la gestión de cada sesión, los elementos significativos que aportan al micro análisis de la Trayectoria Hipotética
Palabras clave: Ambientes de Aprendizaje [AA], Cultura Estadística [CE], Experimento de Enseñanza, Educación Estadística Crítica [EEC], Análisis Exploratorio de Datos [AED], Contexto.

de Aprendizaje y la reformulación del diseño; para finalmente llevar a cabo la Fase 3 (F3) Análisis Retrospectivo, el cual profundiza en el análisis de la situación de enseñanza a través de la contrastación entre metas de aprendizaje y los alcances del Diseño Instruccional, con base en lo cual se plantea un modelo teórico.

\section{Conclusiones}

A partir del diseño, implementación y caracterización de los Ambientes de Aprendizaje que suscitan el desarrollo y apropiación de la Cultura Estadística, se logra identificar que: I) es necesario la caracterización del contexto de la clase, con el fin de establecer condiciones iniciales para los Ambientes de Aprendizaje que propician el desarrollo de la Cultura Estadística, II) lo cual se puede lograr a partir de la conjugación de 7 principios: Cognitivo de la Estadística, Razonamiento estadístico, Análisis crítico de los datos, Contextualización de los datos, Comunicación y organización en el aula, Políticas institucionales y Tecnología y productividad; III) dichos principios son considerados como ejes articuladores que permiten orientar las actividades de los estudiantes a partir de un transitar por los Ambientes de Aprendizaje, que son caracterizados, por lo menos, por uno de los principios anteriormente mencionados. 


\section{La argumentación como núcleo de la actividad demostrativa}

Bibiana Patricia Franco Avendaño

Giovanni Moreno Cárdenas

Palabras clave: Argumentación, Actividad demostrativa,

Tipos de argumentos, Dato, Justificación, Conclusión.

\section{Descripción}

En esta investigación se presenta un estudio realizado en un curso de grado octavo de básica secundaria que tuvo lugar en año 2010 de una institución privada de la ciudad de Bogotá. El propósito es presentar evidencias de que las acciones de la actividad demostrativa promueven procesos argumentativos en los estudiantes, además de tipificar los argumentos que utilizan los estudiantes cuando quieren justificar sus afirmaciones, construcciones o estrategias de solución. En este sentido el objetivo general del estudio es construir e implementar situaciones problema que favorezcan la práctica argumentativa en estudiantes de grado octavo en un marco de actividad demostrativa y analizar los argumentos producidos por ellos. La investigación se desarrolló en varios momentos; en primer lugar se planeó y se puso en marcha el experimento de enseñanza, en segundo lugar se tomó registro de la actividad desarrollada por los estudiantes durante los dos últimos problemas del experimento y por último se analizaron los datos recogidos en las transcripciones de los diálogos de los estudiantes.

\section{Contenidos}

- Delimitación del problema En esta sección, se hizo una justificación y formulación del problema, una presentación de los objetivos y una revisión de antecedentes que plasman los referentes teóricos necesarios para el estudio.

- Marco teórico Se consolidaron los tres aspectos que fundamentan el marco teórico que son: acercamiento a una noción de actividad demostrativa, acercamiento a una noción de argumentación y la tipificación de argumentos.

- Metodología Se hizo una presentación del proceso llevado a cabo durante la investigación. Se describió la perspectiva investigativa; la contextualización del estudio en el cual se describen quienes eran los estudiantes y la clase de geometría; el diseño experimental y el dispositivo analítico.

- Análisis y Resultados Se presentó el informe de cada una de las actividades mencionadas en el dispositivo analítico del capítulo de la metodología y que permitieron analizar los datos obtenidos durante la puesta 
en marcha del experimento de enseñanza.

- Conclusiones En este capítulo se enuncian algunas reflexiones finales que tienen en cuenta aspectos como: la fiabilidad de las transcripciones, el diseño e implementación del experimento de enseñanza, las categorías de tipificación de los argumentos y los resultados de la tipificación.

\section{Metodología}

De acuerdo con el problema descrito en el primer capítulo del trabajo, la investigación se llevó a cabo a partir de la construcción e implementación del experimento de enseñanza.

De las 11 situaciones problema que se implementaron, solamente se grabaron en audio y video las dos últimas situaciones y con tan solo dos grupos de estudiantes, esto con el fin de evitar interferencias auditivas. El experimento de enseñanza buscaba favorecer las acciones de la actividad demostrativa y a partir de los datos obtenidos en su desarrollo se procedió al proceso de análisis.

El análisis tuvo en cuenta la caracterización de las acciones de la actividad demostrativa y la tipificación de los argumentos que construimos a partir de la revisión de diferentes estudios concernientes a la categorización de los argumentos utilizados por los estudiantes.

\section{Conclusiones}

Como reflexión final podemos resaltar los siguientes aspectos:

- Es importante contar con unas transcripciones que reflejen fielmente las intervenciones de los estudiantes cuando se enfrentan a una situación problema que promueva la actividad demostrativa.

- El diseño e implementación del experimento de enseñanza fue exitoso en la medida que las intervenciones de los estudiantes recurrían al uso de propiedades y relaciones entre los objetos geométricos involucrados en el problema.

- Las categorías que construimos para tipificar los argumentos de los estudiantes son emergentes de la actividad de investigación realizada, la cual centra su atención en los desarrollos de los estudiantes desde la revisión bibliográfica realizada.

- Los resultados de la tipificación de argumentos fueron sorpresivos debido a que pensábamos que los estudiantes iban a utilizar elementos empíricos para validar sus argumentos, pero en los hallazgos encontrados, los argumentos obedecen en su mayoría al uso de elementos conceptuales, es decir recurren al uso de propiedades y además establecen un razonamiento en sus propuestas. 